\title{
A Reduced Low-Temperature Electro-Thermal Coupled Model for
}

\section{Lithium-ion Batteries}

Jiuchun Jiang ${ }^{\mathrm{a}, \mathrm{b}}$, Haijun Ruan ${ }^{\mathrm{a}, \mathrm{b}}$, Bingxiang Sun ${ }^{\mathrm{a}, \mathrm{b}, 1}$, Weige Zhang ${ }^{\mathrm{a}, \mathrm{b}}$, Wenzhong Gao ${ }^{\mathrm{c}}$, Le Yi Wang ${ }^{\mathrm{d}}$, Linjing Zhang,b

${ }^{a}$ National Active Distribution Network Technology Research Center (NANTEC), Beijing Jiaotong University, Beijing, 100044, China

${ }^{\mathrm{b}}$ Collaborative Innovation Center of Electric Vehicles in Beijing, Beijing Jiaotong University, Beijing, 100044, China

${ }^{c}$ Department of Electrical and Computer Engineering, University of Denver, Denver, CO 80208 USA

${ }^{\mathrm{d}}$ Department of Electrical and Computer Engineering, Wayne State University, Detroit, MI 48202, USA

Abstract - A low-temperature electro-thermal coupled model, which is based on the electrochemical mechanism, is developed to accurately capture both electrical and thermal behaviors of batteries. Activation energies reveal that temperature dependence of resistances is greater than that of capacitances. The influence of frequency on polarization voltage and irreversible heat is discussed, and frequency dependence of polarization resistance and capacitance is obtained. Based on the frequency-dependent equation, a reduced low-temperature electro-thermal coupled model is proposed and experimentally validated under different temperature, frequency and amplitude conditions. Simulation results exhibit good agreement with experimental data, where the maximum relative voltage error and temperature error are below $2.65 \%$ and $1.79^{\circ} \mathrm{C}$, respectively. The reduced model is demonstrated to have almost the same accuracy as the original model and require a lower computational effort. The effectiveness and adaptability of the proposed methodology for model reduction is verified using batteries with three different cathode materials from different manufacturers. The reduced model, thanks to its high accuracy and simplicity, provides a promising candidate for development of rapid internal heating and optimal charging strategies at low temperature, and for evaluation of the state of battery health in on-board battery management system.

Keywords-Lithium-ion battery, low temperature, electro-thermal coupled model, frequency dependence, model reduction

\section{Nomenclature}

$\begin{array}{llll}L & \text { Inductance }(\mathrm{H}) & u_{b} & \text { Voltage of ohmic resistance }(\mathrm{V}) \\ u_{L} & \text { Voltage of inductance }(\mathrm{V}) & R_{f} & \text { Resistance for passive film }(\Omega) \\ R_{b} & \text { Ohmic resistance }(\Omega) & C_{f} & \text { Capacitance for passive film }(\mathrm{F})\end{array}$

\footnotetext{
${ }^{1}$ Corresponding author. Tel: +86-10-5168-3907; fax: +86-10-5168-7101. E-mail: bxsun@bjtu.edu.cn. Electrical engineering building, Beijing Jiaotong University, Beijing, China
} 


\begin{tabular}{|c|c|c|c|}
\hline$u_{f}$ & Voltage of film impedance $(\mathrm{V})$ & $E_{\text {arb }}$ & Activation energy of $R_{b}\left(\mathrm{~kJ} \cdot \mathrm{mol}^{-1}\right)$ \\
\hline \multirow[t]{2}{*}{$R_{a}$} & Charge-transfer resistance on the anode & $E_{a c f}$ & Activation energy of $C_{f}\left(\mathrm{~kJ} \cdot \mathrm{mol}^{-1}\right)$ \\
\hline & surface $(\Omega)$ & $E_{\text {aca }}$ & Activation energy of $C_{a}\left(\mathrm{~kJ} \cdot \mathrm{mol}^{-1}\right)$ \\
\hline \multirow[t]{2}{*}{$C_{a}$} & Charge-transfer capacitance on the anode & $E_{a c c}$ & Activation energy of $C_{c}\left(\mathrm{~kJ} \cdot \mathrm{mol}^{-1}\right)$ \\
\hline & surface $(F)$ & $E_{a c d}$ & Activation energy of $C_{d 1}$ and $C_{d 2}$ \\
\hline \multirow[t]{2}{*}{$u_{a}$} & Charge-transfer voltage on the anode & & $\left(\mathrm{kJ} \cdot \mathrm{mol}^{-1}\right)$ \\
\hline & surface $(V)$ & $u_{b L}$ & Total voltage of $\mathrm{R}_{\mathrm{b}}$ and $\mathrm{L}(\mathrm{V})$ \\
\hline \multirow[t]{2}{*}{$R_{c}$} & Charge-transfer resistance on the cathode & $Q_{f}$ & Heat generation of $R_{f}(\mathrm{~J})$ \\
\hline & surface $(\Omega)$ & $Q_{a}$ & Heat generation of $R_{a}(\mathrm{~J})$ \\
\hline \multirow[t]{2}{*}{$C_{c}$} & Charge-transfer capacitance on the & $Q_{c}$ & Heat generation of $R_{c}(\mathrm{~J})$ \\
\hline & cathode surface $(\mathrm{F})$ & $Q_{d 1}$ & Heat generation of $R_{d l}(\mathrm{~J})$ \\
\hline \multirow[t]{2}{*}{$u_{c}$} & Charge-transfer voltage on the cathode & $Q_{d 2}$ & Heat generation of $R_{d 2}(\mathrm{~J})$ \\
\hline & surface $(\mathrm{V})$ & $Q_{b}$ & Heat generation of $R_{b}(\mathrm{~J})$ \\
\hline$R_{d 1}$ & Resistance of diffusion process $(\Omega)$ & $\mathrm{Z}_{\mathrm{p}}$ & Polarization resistance $(\Omega)$ or capacitance \\
\hline$R_{d 2}$ & Resistance of diffusion process $(\Omega)$ & & (F) \\
\hline$C_{d 1}$ & Capacitance of diffusion process $(\mathrm{F})$ & $\mathrm{Z}_{\alpha}$ & Parameter of polarization resistance $(\Omega)$ \\
\hline$C_{d 2}$ & Capacitance of diffusion process $(\mathrm{F})$ & & or capacitance $(\mathrm{F})$ \\
\hline$u_{d 1}$ & Voltage of diffusion process $(\mathrm{V})$ & $\mathrm{Z}_{\beta}$ & Parameter of polarization resistance $(\Omega)$ \\
\hline$u_{d 2}$ & Voltage of diffusion process $(\mathrm{V})$ & & or capacitance $(\mathrm{F})$ \\
\hline$i$ & Current through the battery (A) & $\mathrm{Z}_{\gamma}$ & Parameter of polarization resistance $(\Omega)$ \\
\hline$C_{p}$ & Polarization capacitance (F) & & or capacitance $(\mathrm{F})$ \\
\hline$R_{p}$ & Polarization resistance $(\Omega)$ & Abbrev & tions \\
\hline$u_{o}(\mathrm{t})$ & Terminal voltage (V) & BMS & Battery management system \\
\hline$u_{p}(\mathrm{t})$ & Total polarization voltage $(\mathrm{V})$ & $\mathrm{C}$ & Capacity rate \\
\hline$U_{o c v}$ & Open circuit voltage $(\mathrm{V})$ & $\mathrm{CFCC}$ & Constant frequency constant current \\
\hline$R$ & Gas constant $\left(\mathrm{J} \cdot \mathrm{mol}^{-1} \cdot \mathrm{K}^{-1}\right)$ & CFVC & Constant frequency variable current \\
\hline$T$ & Battery temperature $(\mathrm{K})$ & DC & Direct current \\
\hline$A_{1}$ & Pre-exponential factor & EEC & Equivalent electrical circuit \\
\hline$k$ & Reaction rate constant $\left(\mathrm{mol} \cdot \mathrm{m}^{3} \cdot \mathrm{s}^{-1}\right)$ & EIS & Electrochemical impedance spectroscopy \\
\hline$E_{a}$ & Activation energy $\left(\mathrm{kJ} \cdot \mathrm{mol}^{-1}\right)$ & ETC & Electro-thermal coupled \\
\hline$H_{t o t}$ & The total enthalpies $(\mathrm{J})$ & $\mathrm{EV}$ & Electric vehicle \\
\hline$q$ & Heat transfer rate with surroundings (W) & EW & Electrochemical workstation \\
\hline$P_{a}$ & Active power of electrical work (W) & FD & Frequency-dependent \\
\hline$T_{a}$ & Ambient temperature $\left({ }^{\circ} \mathrm{C}\right)$ & FFT & Fast Fourier Transformation \\
\hline$A$ & Surface area of the battery $\left(\mathrm{m}^{2}\right)$ & LCO & $\mathrm{LiCoO}_{2}$ \\
\hline$h$ & Heat-transfer coefficient $\left(\mathrm{W} \cdot \mathrm{m}^{-2} \cdot \mathrm{K}^{-1}\right)$ & LFP & $\mathrm{LiFePO}_{4}$ \\
\hline$m$ & Battery mass (g) & LIB & Lithium-ion battery \\
\hline$C_{p}^{\prime}$ & Specific heat capacity $\left(\mathrm{J} \cdot \mathrm{g}^{-1} \cdot \mathrm{K}^{-1}\right)$ & $\mathrm{NCM}$ & $\mathrm{LiNi}_{1 / 3} \mathrm{Co}_{1 / 3} \mathrm{Mn}_{1 / 3} \mathrm{O}_{2}$ \\
\hline$U$ & Potential of the battery $(\mathrm{V})$ & $\mathrm{RC}$ & Resistance and capacitance \\
\hline$\theta$ & Phase angle (rad) & REEC & Reduced equivalent electrical circuit \\
\hline$E_{a r f}$ & Activation energy of $R_{f}\left(\mathrm{~kJ} \cdot \mathrm{mol}^{-1}\right)$ & RETC & Reduced electro-thermal coupled \\
\hline$E_{\text {ara }}$ & Activation energy of $R_{a}\left(\mathrm{~kJ} \cdot \mathrm{mol}^{-1}\right)$ & RQ & Resistance and constant phase element \\
\hline$E_{a r c}$ & Activation energy of $R_{c}\left(\mathrm{~kJ} \cdot \mathrm{mol}^{-1}\right)$ & SAC & Sinusoidal alternating current \\
\hline$E_{\text {ard }}$ & Activation energy of $R_{d 1}$ and $R_{d 2}$ & SOC & State of charge \\
\hline & $\left(\mathrm{kJ} \cdot \mathrm{mol}^{-1}\right)$ & UDDS & Urban Dynamometer Driving Schedule \\
\hline
\end{tabular}




\section{Introduction}

The lithium-ion battery (LIB), thanks to its high cell voltage, high energy density, long lifetime, and environmental friendliness, is regarded as a favorable candidate for electric vehicles (EVs) [1]. However, the dramatically increased impedance at low temperature substantially reduces the pulse power and driving range of EVs [2]. More severely, Li deposition, which is easily formed through an unwanted side reaction during charging at low temperatures [3], affects battery lifetime dramatically and even results in safety hazards, such as thermal runaway. The key to battery performance improvement is to establish a suitable model and manage the temperature properly. Generally speaking, LIBs need to be preheated before charging or discharging in cold weather until the batteries reach a performance-friendly temperature. Internal heating can help achieve better uniformity of the temperature distribution in LIBs with higher efficiency in contrast to external heating [4]. To seek an optimal internal heating strategy, it is critical to establish the thermal model to predict the temperature evolution within LIBs. Since the heat is generated from electrical processes [5, 6], and rapid and safe low-temperature charging is expected [7], it is essential to build a high-fidelity model for voltage estimation of LIBs.

There are several modeling strategies, such as the black box model, the electrochemical model and the equivalent electrical circuit model, to predict voltage performance of LIBs. The black box model, predicting the terminal voltage of LIBs using mathematical algorithms [8] such as artificial neural network [9], is difficult for exploring internal characteristics of LIBs. In addition, the accuracy of voltage estimation is highly dependent on the trained data, resulting in its limited adaptability. Although it can characterize the reaction mechanism inside the battery [10], the electrochemical model, containing complicated nonlinear partial differential equations with many unknown variables, requires significantly long computation time and high parameterization effort [11]. The equivalent electrical circuit model, representing the equivalent form of the electrochemical model by using electrical circuit elements [12-15], can explicitly characterize the performance of LIBs with a good compromise between computation time, parameterization effort, and simulation accuracy $[16,17]$.

Two equivalent electrical circuit models, namely, one with RQ and Warburg elements, and the other with RC-elements, are presented to predict the LIB performance based on electrochemical impedance spectroscopy (EIS) data [18, 19]. Although RQ and Warburg elements can characterize the electrochemical performance of LIBS well, their Laplace transforms are very complicated [20], which makes them difficult for application in the time-domain. Furthermore, they significantly increase the 
computational complexity and computation time, leading to difficulty for describing the current-voltage characteristic of LIBs [21]. On the other hand, the equivalent electrical circuit model, in which RC-elements are derived from measured EIS data in the frequency-domain [22,23], is used to capture the current-voltage performance in real-time with low computational effort.

A general equivalent electrical circuit model shows a lower accuracy at low temperature [24]. Sluggish charge-transfer kinetics, decreased solid-state lithium-ion diffusivity and reduced electrolyte conductivity within LIBs are found at low temperature. Furthermore, there exists strong mutual coupling of a variety of influence factors within LIBs at low temperature. So the low-temperature modeling of LIBs is challenging due to the high nonlinear characteristic of model parameters compared to room-temperature modelling [25]. On the other hand, the low-temperature performance of LIBs is complicated and model parameters are sensitive to temperature. Most models, which are developed to describe room or elevated temperature performance of LIBs, always describe low-temperature performance in a complicated way or are not even suitable to capture the behavior of LIBs at low temperature [26]. To describe the low-temperature performance of LIBs, a complex mathematical formula considering the effect of the temperature is introduced for battery modelling in [25]. Moreover, only the discharge behavior is included in the model in [25]. In the electrochemical model presented in [24], nonlinear differential equations with many unknown variables make the model complicated. Although the model in [26] is simplified, there are still many undetermined parameters, and the current used to validate for the reduced model is much lower. Moreover, the model only describes the charge behavior under low current. Therefore, developing a simplified and highly accurate model, considering the effect of the temperature and the SAC frequency with high current under the charging and discharging operating conditions, is essential for batteries' applications in cold weather.

In this work, a novel reduced low-temperature electro-thermal coupled model is proposed with the following advantages. First, a novel frequency-dependent equation for polarization parameters is presented. So the sophisticated model is simplified as a reduced model. Second, the reduced model is experimentally validated under different frequency and low-temperature conditions. Simulation results from the model show good agreement with experimental data, where the maximum relative voltage error and temperature error are less than $2.65 \%$ and $1.79^{\circ} \mathrm{C}$, respectively. Third, this new model is demonstrated to have almost same accuracy as the original sophisticated model and yet require a lower computational effort. Last but not least, the effectiveness and adaptability of the 
proposed methodology for model reduction is verified using batteries with three different cathode materials from different manufacturers. The reduced model, thanks to its high accuracy and simplicity, yields a promising candidate for development of the rapid internal heating and optimal charging strategies at low temperature, and for evaluation of the state of battery health in on-board battery management system (BMS).

The remaining part of this paper is structured as follows. The experimental setup and procedures are briefly described in Section 2. The electro-thermal coupled model is developed in Section 3, while the model simplification process is explained in detail in Section 4. Model verification results and discussions are presented in Section 5. Finally, Section 6 gives a conclusion.

\section{Experimental}

\subsection{Batteries and Test Workbench}

For simplicity but without loss of generality, the commercial 18650 battery as shown in Table 1 , consisting of graphite anode material and $\mathrm{LiNi}_{1 / 3} \mathrm{Co}_{1 / 3} \mathrm{Mn}_{1 / 3} \mathrm{O}_{2}(\mathrm{NCM})$ cathode material, is used for all experiments, whose weight is $45 \mathrm{~g}$. Other two batteries with common graphite anode materials but differentiated cathode materials, namely the $\mathrm{LiFePO}_{4}$ (LFP) battery and the $\mathrm{LiCoO}_{2}(\mathrm{LCO})$ battery, are only used for electrical experiments at $-15^{\circ} \mathrm{C}$.

Table 1. Batteries used for tests

\begin{tabular}{ccccc}
\hline Battery & Cathode material & Nominal capacity(Ah) & Cut-off voltages(V) & Manufacturer \\
\hline INR18650-29E & $\mathrm{LiNi}_{1 / 3} \mathrm{Co}_{1 / 3} \mathrm{Mn}_{1 / 3} \mathrm{O}_{2}$ & 2.75 & $4.2 / 2.5$ & Samsung SDI \\
IFR18650P-1100 & $\mathrm{LiFePO}_{4}$ & 1.1 & $3.65 / 2.0$ & New TaiHang \\
\hline $\mathrm{LiCoO}_{2}$ & 2.5 & $4.2 / 3.0$ & LISHEN \\
\hline & & & \\
& &
\end{tabular}

Fig. 1. Experimental platform for battery tests.

Fig. 1 exhibits the experimental platform for battery tests, which comprises of the testing system and monitor system. The testing system consists of an electrochemical workstation (EW) carrying out EIS measurements and SAC excitation, an external potentiostat PP241 applied to amplify current, a climate chamber providing controllable temperature, an incubator, and experimental batteries. The monitor system, monitoring and logging voltage, current and temperature data, contains a personal 
computer, a current probe, a digital oscilloscope and a data acquisition unit.

\subsection{EIS Measurements}

EIS measurements were conducted using EW over a temperature range from $-15^{\circ} \mathrm{C}$ to $6^{\circ} \mathrm{C}$ with intervals of $3^{\circ} \mathrm{C}$ at $50 \%$ SOC. The battery was soaked at each temperature in the incubator within the climate chamber for more than $5 \mathrm{~h}$ for thermal equilibration. EIS experimental conditions were: perturbation current, $I_{a}=200 \mathrm{~mA}$ over the frequency range from $10 \mathrm{mHz}$ to $10 \mathrm{kHz}$, for eight points per decade above $66 \mathrm{~Hz}$ and four points per decade below $66 \mathrm{~Hz}$. LFP and LCO batteries experience the same EIS measurements at $-15^{\circ} \mathrm{C}$.

\subsection{Model Verification Experiments}

Verification experiments for voltage performance were performed using SAC with various sets of frequencies, temperatures and amplitudes, and using the dynamically varying current profile, such as the UDDS profile for NCM batteries as shown in Table 2. Verification experiments for thermal behavior were carried out under different SAC conditions including constant frequency constant current (CFCC) and constant frequency variable current (CFVC) as shown in Table 2. The battery was kept in the incubator at different temperatures, such as $-15^{\circ} \mathrm{C}$, for more than $5 \mathrm{~h}$ before testing. Battery temperatures were measured using five T-type thermocouples, the average of which is defined as the experimental temperature of LIBs. As to LFP and LCO batteries, verification experiments for electrical behavior were carried out using SAC with various sets of frequencies and amplitudes at $-15^{\circ} \mathrm{C}$.

Table 2. Experimental details.

\begin{tabular}{ccc}
\hline Test & Item & Value \\
\hline & Different frequencies & $50 \mathrm{~Hz}, 500 \mathrm{~Hz}, 5 \mathrm{kHz}$ \\
Different temperatures & $-15^{\circ} \mathrm{C},-10^{\circ} \mathrm{C},-5^{\circ} \mathrm{C}, 0.3^{\circ} \mathrm{C}$ \\
Validation & Different amplitudes & $10 \mathrm{~A}, 2 \mathrm{~A}, 1 \mathrm{~A} 0.5 \mathrm{~A}, 0.2 \mathrm{~A}$ \\
tests & CFCC & $50 \mathrm{~Hz}, 5 \mathrm{~A} ; 500 \mathrm{~Hz}, 10 \mathrm{~A} ; 5 \mathrm{kHz}, 10 \mathrm{~A}$ \\
& CFVC & $500 \mathrm{~Hz}-5,10,20 \mathrm{~A} ; 5 \mathrm{kHz}-5,10,13.3 \mathrm{~A}$ \\
& Dynamically varying current profile & The UDDS profile \\
\hline
\end{tabular}

\section{Electro-thermal Coupled Model}

\subsection{Electrical Model}

An electrochemical mechanism based equivalent electrical circuit (EEC) model as shown in Fig. 2 is presented to outline low-temperature current-voltage behavior of LIBs, whose parameters are independent of the SAC frequency. The Laplace transform for RQ-elements as well as Warburg elements is very complicated [27] when applied in the time-domain. Approximations using 
RC-elements are derived, which significantly decrease the computational complexity and effort. Two RC-elements $\left(R_{c}, C_{c}\right.$ and $\left.R_{a}, C_{a}\right)$ are adopted to describe the kinetic processes of charge-transfer on the cathode and anode surfaces [28], respectively. Other two RC-elements $\left(R_{d 1}, C_{d 1}\right.$ and $\left.R_{d 2}, C_{d 2}\right)$ are analogously employed to describe lithium-ion diffusivity in the solid state. Furthermore, an RC-element $\left(R_{f}, C_{f}\right)$ represents Li-ion migration through the passive film while an RL-element $\left(R_{b}, L\right)$ indicates the response of LIBs at high frequency [29]. Thereby, the EEC model contains all information about the battery performance by equivalent RLC-elements, which can accurately capture the electrical behavior.

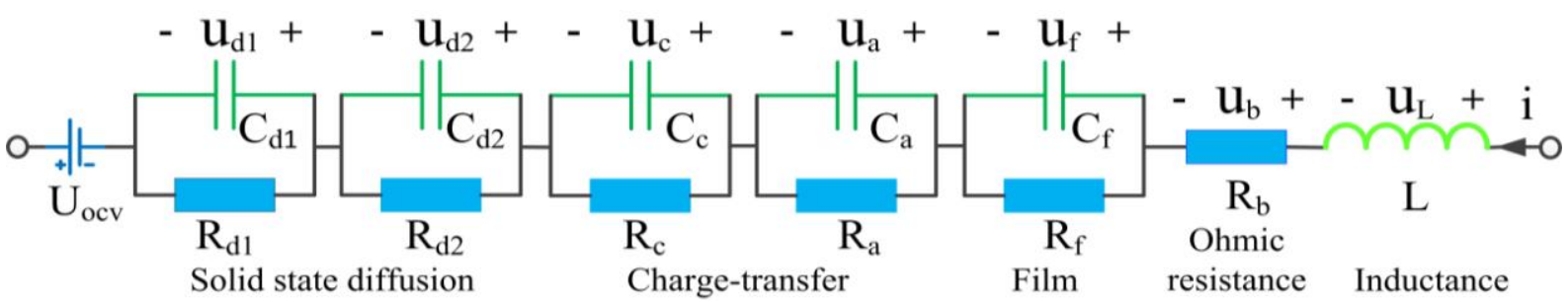

Fig.2. Electrochemical mechanism based equivalent electrical circuit model

The variation of $\ln (k)$ as a linear function of the reciprocal of temperature is in agreement with Arrhenius behavior [30] described by

$$
k=A_{1} \times \mathrm{e}^{-\frac{E_{a}}{R T}}
$$

The activation energy embodies the impact of temperature on the reaction rate. Higher activation energy implies greater improvement of the reaction rate when the battery temperature rises. The reaction rate constant $(k)$ has nothing to do with the concentration of the reactants. It is dependent on the current density in the electrochemical system. On the other hand, the resistance is inversely proportional to the current density and the capacitance is proportional to the current density under the condition of a certain constant voltage. Consequently

$$
\left\{\begin{array}{l}
\ln \left[R_{x}(T)\right]=\frac{E_{a r x}}{1000 \times R} \times \frac{1000}{T}+\ln b_{r x} \\
\ln \left[C_{x}(T)\right]=-\frac{E_{a c x}}{1000 \times R} \times \frac{1000}{T}+\ln b_{c x}
\end{array}\right.
$$

where $R_{x}(T)$ and $C_{x}(T)$ represent the resistance and the capacitance in the prominent electrochemical processes, respectively. $b_{1 x}$ and $b_{2 x}$ stand for the proportional coefficients.

\subsection{Thermal Model}

The battery temperature is assumed to be uniform and a lumped thermal model is developed. The improved equation (3) can be deduced from the first law of thermodynamics [31].

$$
\frac{d H_{t o t}}{d t}=q+P_{a}
$$


The rate of heat transfer between the battery and surroundings is based on the well-known Newton cooling law [32]. If an average heat capacity is adopted, Eq. (3) can be written as

$$
m C_{p}^{\prime} \frac{d T}{d t}=P_{a}-I T \frac{d U}{d T}-h A\left(T-T_{a i r}\right)
$$

The equivalent heat-transfer coefficient is estimated from the heat losses from the battery, which characterizes the heat transfer/exchange effect between the battery and the environment. The second item on the right hand side of the equation represents the reversible heat, which is related to the entropy change within LIBs. When the battery is subjected to a symmetrical alternating current, the reversible heat generated during a period of SAC is nearly zero. Therefore

$$
m C_{p}^{\prime} \frac{d T}{d t}=I V \cos \theta-h A\left(T-T_{\text {air }}\right)
$$

The phase angle is the angle between the sinusoidal alternating voltage and SAC. The first item on the right hand side of the equation, representing the irreversible heat, is composed of heat generated from ohmic loss, film hindrance, charge-transfer over-potential, and mass transfer limitation. The specific heat capacity and the equivalent heat-transfer coefficient can be estimated based on the method presented in the literature [33].

\subsection{Electro-Thermal Coupled Model}

\subsubsection{Modeling}

The electrochemical mechanism based low-temperature electro-thermal coupled (ETC) model, which is shown in Fig.3, combines the EEC model and the thermal model. The coupling of thermal and electrical models arises from highly temperature-dependent kinetic and transport properties, and the heat generated from the electrical behavior. When the battery is subjected to SAC excitation, the terminal voltages can be calculated based on the EEC model. Accordingly, the heat generated from electrical process, namely the active power $P_{a}$, dictates battery temperature evolution. On the other hand, the battery temperature affects the electrical process because of temperature-dependent parameters. RC-elements, namely $Z(T)$, vary with the temperature based on Eq.(2). Next, the parameters that change with temperature affect the voltage profiles from the EEC model. Then the active power will vary, which has an impact on the heat generation rate and the temperature-rise rate. In short, there exist strong mutual coupling between electrical and thermal processes during the operation of LIBs. Electro-thermal coupled modeling is critical to the prediction of the battery behavior, including the terminal voltage and the battery temperature. 


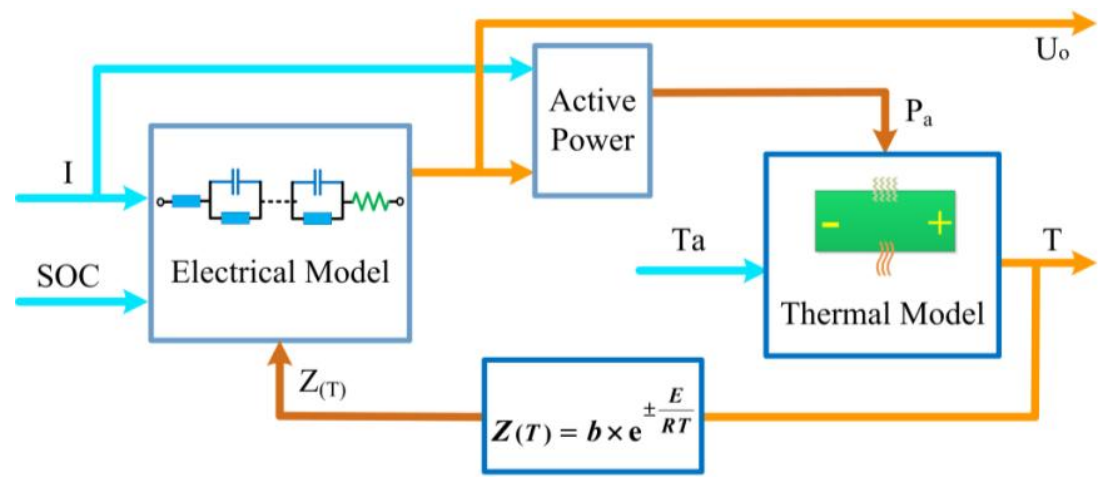

Fig.3. Electro-thermal coupled model

\subsubsection{Model Extraction}

Parameters of the presented EEC model at different temperatures are fitted using the EIS data through the SIM software and only the inductance is independent of temperature. Fig. 4 shows that the natural logarithm of both resistances and capacitances in prominent electrochemical processes varies linearly with the reciprocal of the absolute temperature. The resistances increase while capacitances decrease when the temperature goes down. They follow nicely Eq. (2), which shows that the model fitting for varying temperature works consistently.
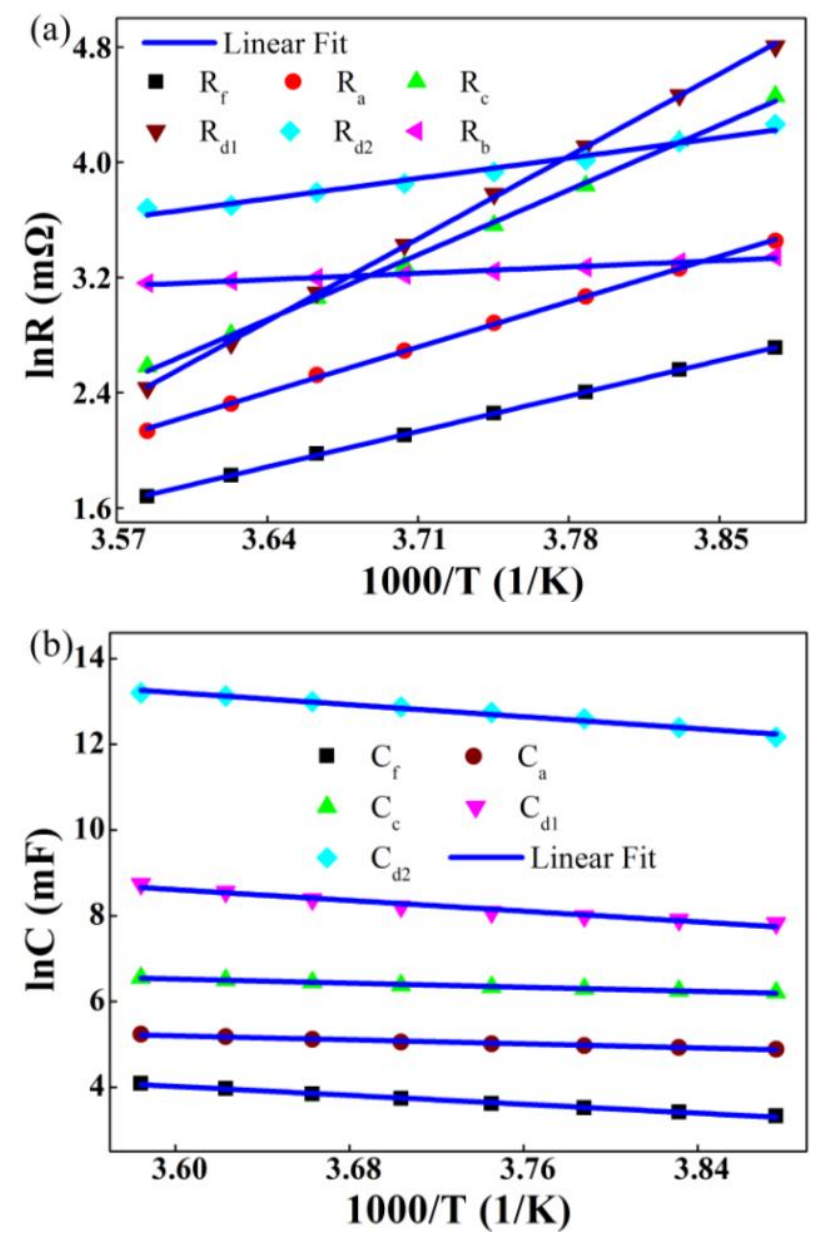

Fig.4. Temperature dependence (a) Resistances (b) Capacitances

Activation energies of resistances are mostly greater than that of capacitances as shown in Table 
3 , implying that temperature dependence of resistances is greater. Activation energies of chargetransfer resistances are large, indicating the profound impact of temperature on the Li-ion intercalation and de-intercalation. Generally, the charge-transfer resistances, which reflect the electrochemical reaction rate, substantially increase with the decreasing temperature, becoming the dominant part of the battery impedance at low temperature. The activation energy of ohmic resistance is relatively small, which indicates that the electronic and ionic conductivities are less sensitive to temperature.

Table 3.activation energies of prominent electrochemical processes.

\begin{tabular}{|c|c|c|c|c|c|}
\hline & $E_{a r f}$ & $E_{\text {ara }}$ & $E_{\text {arc }}$ & $E_{\text {ard }}$ & $E_{a r b}$ \\
\hline Activation Energy & 29.2 & 37.4 & 53.4 & 38.4 & 5.2 \\
\hline \multirow[t]{2}{*}{$\left(\mathrm{kJ} \cdot \mathrm{mol}^{-1}\right)$} & $E_{a c f}$ & $E_{a c a}$ & $E_{a c c}$ & $E_{a c d}$ & - \\
\hline & 21.6 & 9.8 & 9.9 & 29.1 & - \\
\hline
\end{tabular}

\section{Model Reduction}

\subsection{Influence of Frequency on Polarization Voltage}

Polarization voltage evolution of some electrochemical reactions under $-15^{\circ} \mathrm{C}$ at several $\mathrm{SAC}$ frequencies, which is based on the presented ETC model, is shown in Fig. 5, where the SAC amplitude is kept the same. The polarization voltage of charge-transfer processes lags the input current and is high at $50 \mathrm{~Hz}$. However, the charge-transfer polarization voltage is extremely small at high frequency, implying the high frequency can restrain the charge-transfer reaction. Despite the very low temperature of LIBs, the possibility of Li plating is substantially minimal with a suitable current at high frequency because the faraday current is almost zero. Polarization voltages of all RC-elements decrease while the inductive polarization voltage, leading the input SAC, increases with the growing frequency. Therefore, the intense suppression of the hindrance in most electrochemical processes can be achieved in high frequency region, which refrains from the most processes inducing performance degradation at low temperature. The phenomenon, the inductive behavior shown at high frequency and capacitive behavior shown at low frequency, indicates that different electrochemical processes can be controlled by varying the SAC frequency.

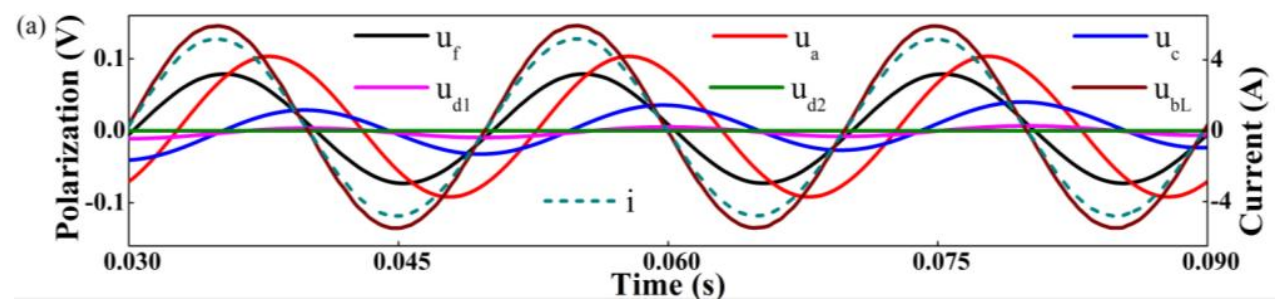



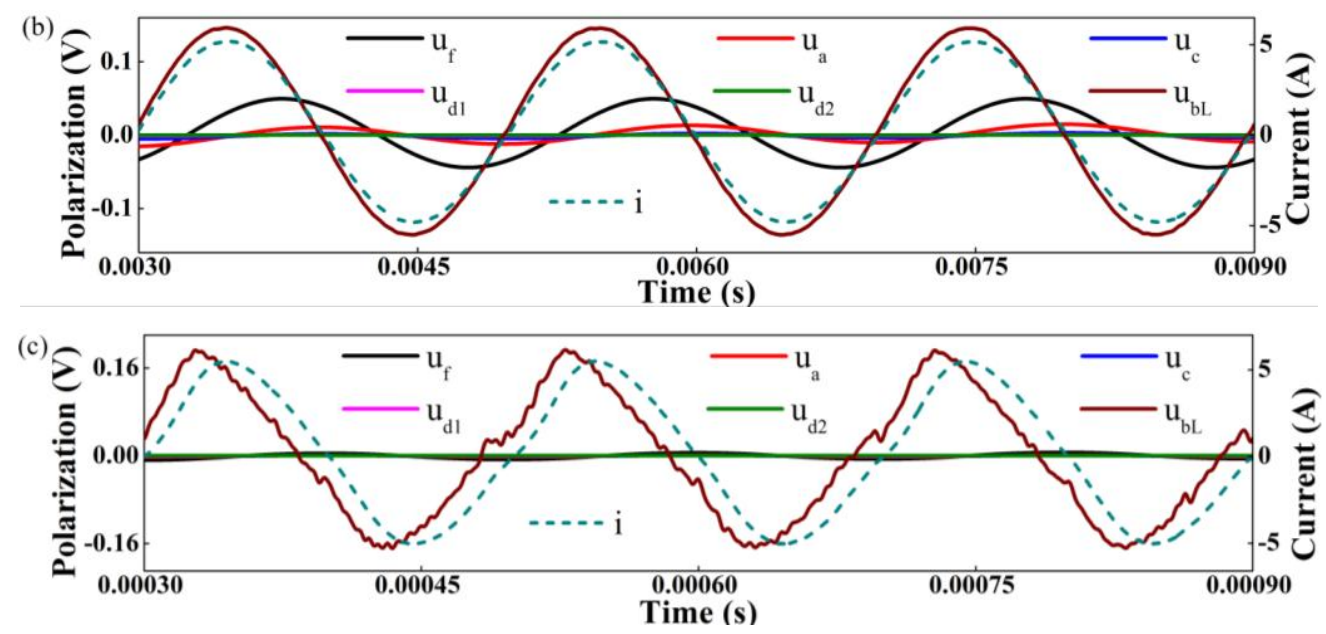

Fig.5. Polarization voltage evolution at (a) $50 \mathrm{~Hz}$,(b) $500 \mathrm{~Hz}$,(c) $5 \mathrm{kHz}$.

\subsection{Influence of Frequency on Irreversible Heat}

Input SAC frequency can also exert a great influence on irreversible heat as shown in Fig.6. Consistent with the situation of polarization voltage, half of the heat is generated from ohmic impedance when the frequency is $50 \mathrm{~Hz}$ while the heat almost completely comes from ohmic impedance at the frequency of $5 \mathrm{kHz}$. The heat from $\mathrm{RC}$-elements gradually decreases with the increasing frequency and the heat from diffusion is virtually zero, which can be neglected when the frequency is above $50 \mathrm{~Hz}$. Apparently, irreversible heat diminishes gradually under the condition of the same current when the frequency goes up due to the decrease of real impedance. Consequently, the irreversible heat within the battery can be controlled by varying the frequency.

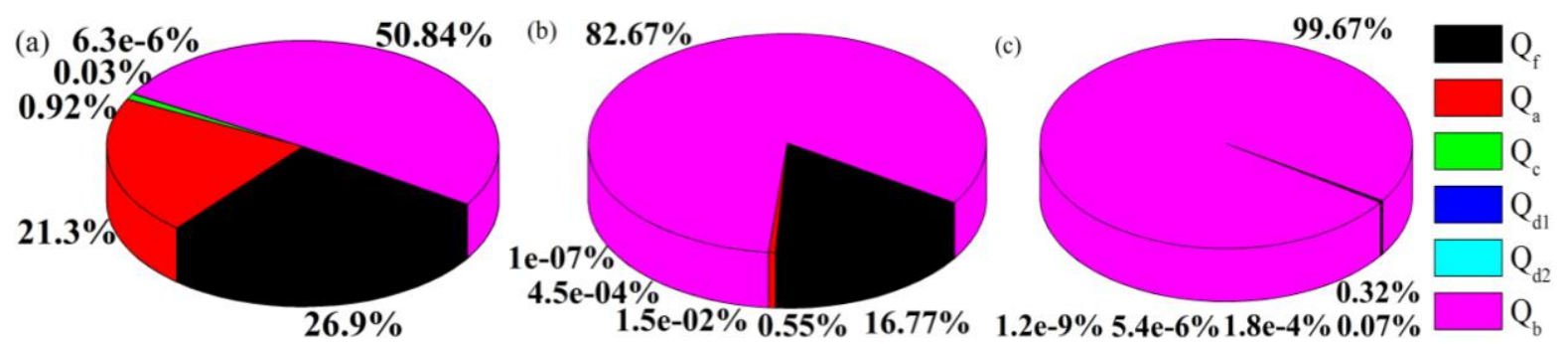

Fig.6. Irreversible heat distribution at (a) $50 \mathrm{~Hz}$,(b) $500 \mathrm{~Hz}$, (c) $5 \mathrm{kHz}$.

\subsection{Frequency Dependence of the Resistance and Capacitance}

The cases, where the polarization voltage and irreversible heat vary with SAC frequency, essentially embody the characteristic of polarization impedance including resistance and capacitance. In fact, the five RC-elements, which characterize different time constants, are equivalent to prominent electrochemical processes in different frequency ranges. Thereby, as long as the RC-element is dependent on frequency, the five RC-elements can be simplified as one RC-element, which can significantly reduce the model. 

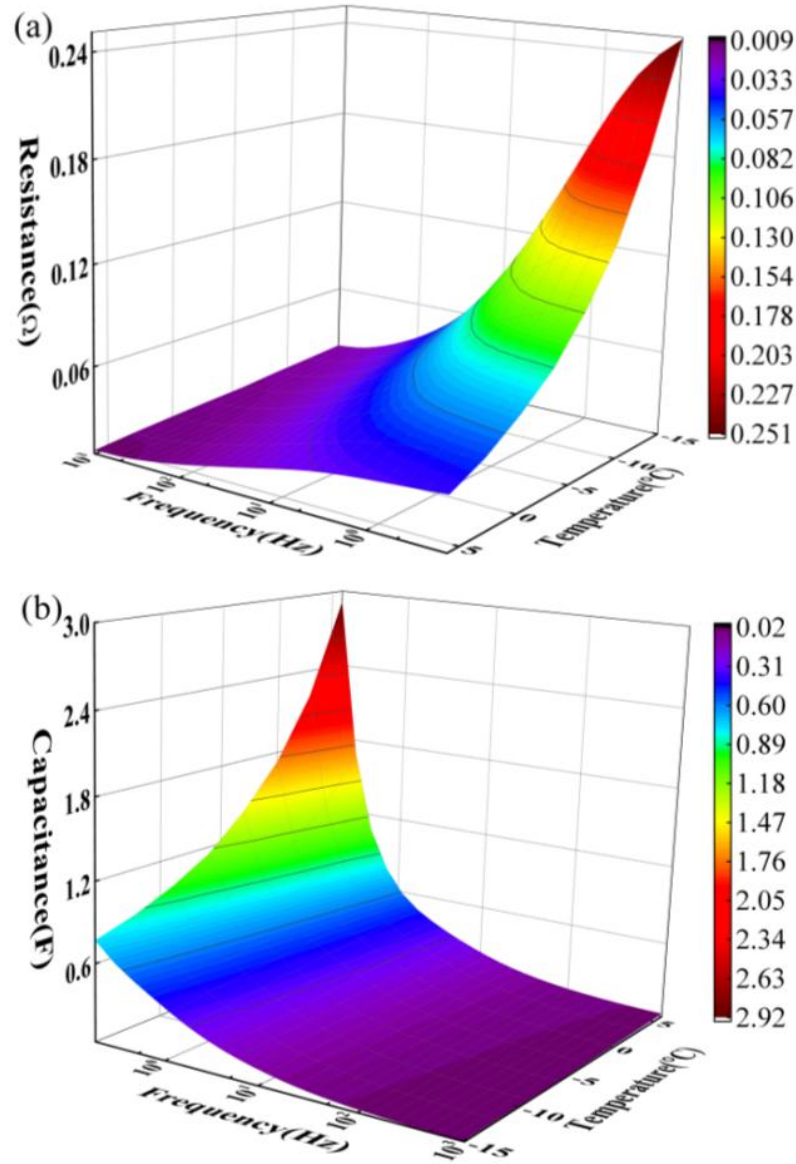

Fig.7. Evolution of (a) resistance, (b) capacitance at different frequencies and temperatures.

The resistance increases while the capacitance diminishes as shown in Fig.7, when the environment temperature decreases. Furthermore, the lower the frequency is, the greater both the resistance and capacitance are. Among all the electrochemical processes, only Warburg impedance for diffusion is dependent on frequency and it is proportional to the square root of the reciprocal of frequency $[34,35]$. For simplicity, the impedance can be governed by Eq. (6), namely frequencydependent (FD) equation, where $Z_{\alpha}, Z_{\beta}$ and $Z_{\gamma}$ are temperature-dependent variables needed to be solved.

$$
Z_{p}=\frac{Z_{\alpha}}{Z_{\beta}+f}+Z_{\gamma}
$$

\subsection{Model Reduction}

A first-order low-temperature model, reduced equivalent electrical circuit (REEC) model as illustrated in Fig.8, is simplified from the original sophisticated fifth-order EEC model. The values of polarization resistance and polarization capacitance vary with frequency based on the FD equation. Parameters of polarization resistance and capacitance in the presented REEC model at different temperatures are fitted by Eq.(6). Then, the relationship between these determined parameters and temperature can be obtained by Eq.(2). The ohmic resistance can be obtained from the real part of 
impedance while the inductance can be calculated from the imaginary part of impedance at high frequency. Based on the REEC model and the thermal model, a simplified low-temperature model, reduced electro-thermal coupled (RETC) model, is proposed to provide information of both electrical and thermal behaviors for application in on-board BMS.

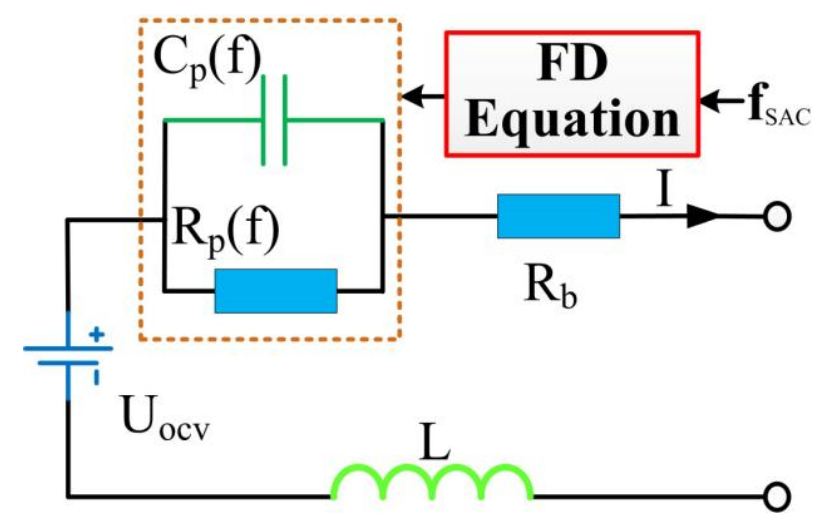

Fig.8 Reduced equivalent electrical circuit model.

The natural logarithm of fitting parameters, which are from fitting the polarization resistance and polarization capacitance based on the FD equation, varies linearly with the reciprocal of the absolute temperature as shown in Fig.9. The slope of resistance parameters is almost larger than that of capacitance parameters, implying that the polarization resistance is more sensitive to temperature. The RL-element can be fitted in the same way as that of the ETC model.

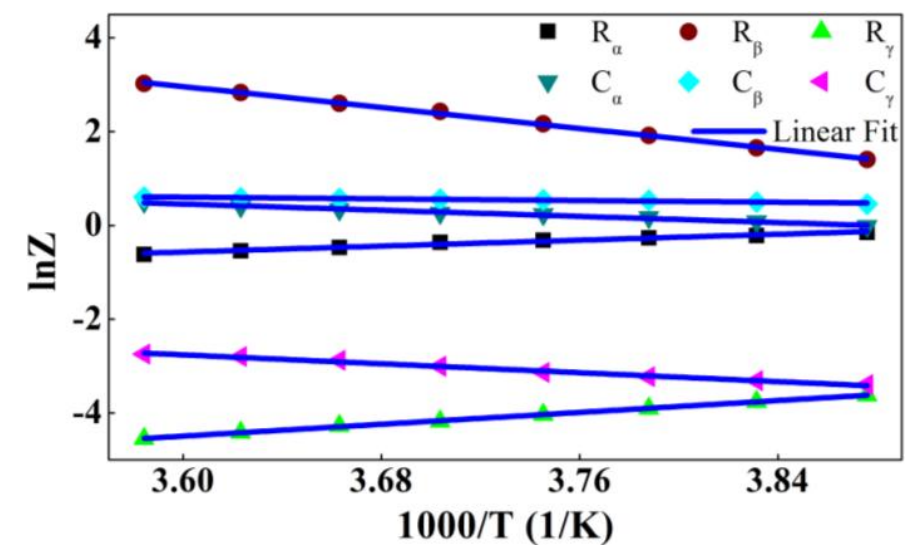

Fig.9.Temperature dependence of the resistance and capacitance parameters.

\section{Results and Discussions}

\subsection{Verification at Different Frequencies}

The validations were carried out with the steady-state SAC excitation at different frequencies under $-15^{\circ} \mathrm{C}$, where the $\mathrm{SAC}$ amplitude is $5 \mathrm{~A}$. Voltage results at $50 \mathrm{~Hz}$ for measurement and simulation are shown in Fig.10 (a). It is observed that presented models can predict the battery voltage with a high accuracy. 

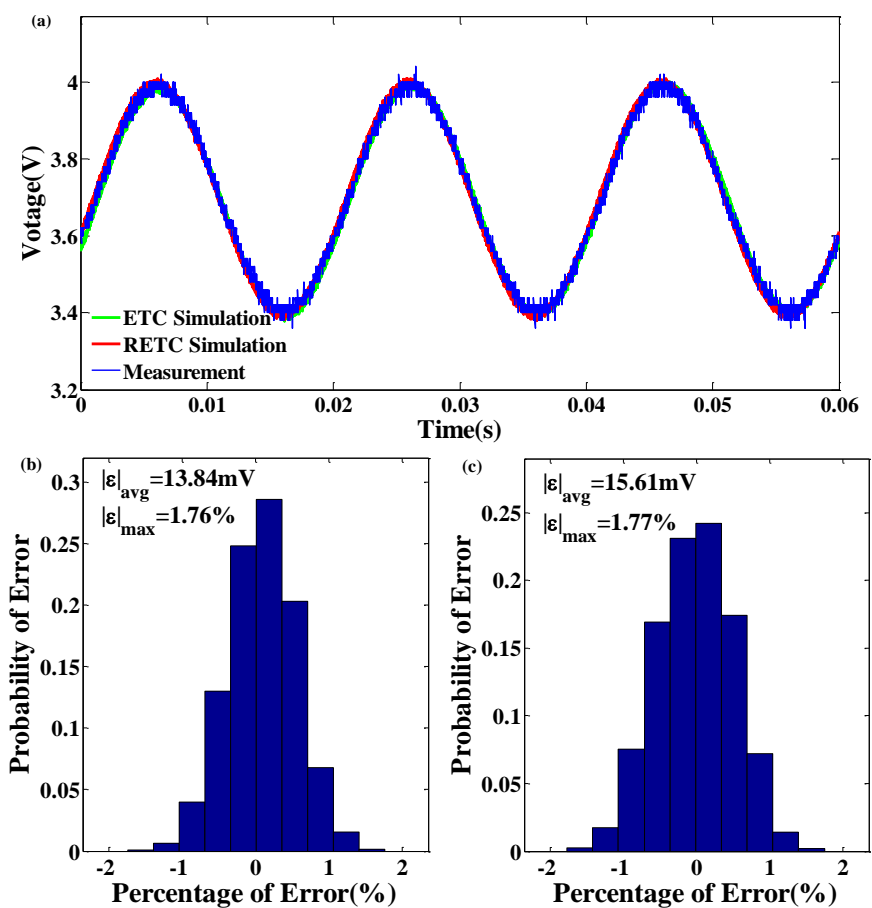

Fig.10. (a) Voltage evolution (b) Error distribution of ETC model simulation (c) Error distribution of RETC model simulation.

Moreover, in order to compare the results, the mean value $|\varepsilon|_{\text {avg }}$ of the absolute error between measured and simulated voltage of LIBs is given, as well as the maximum relative error $|\varepsilon|_{\max }$, which is the ratio between the maximum absolute error and $U_{o c v}$. Two models show a similar distribution of voltage deviations as shown in Fig. 10 (b) and (c). Table 4 lists simulation errors of two models at various frequencies. It is concluded that simulation errors of ETC model are smaller than that of RETC model. However, they have a very comparable performance with mean errors of less than $13.8 \mathrm{mV}$ and $15.6 \mathrm{mV}$, respectively, and maximum relative errors of less than $1.76 \%$ and $1.77 \%$, respectively. The mean errors and maximum relative errors of two models are slightly different at various frequencies but they are all very small, implying that presented models can accurately emulate the voltage behavior of LIBs.

Table 4. Simulation errors of two models at various frequencies.

\begin{tabular}{ccccc}
\hline & Frequency $(\mathrm{Hz})$ & 50 & 500 & 5000 \\
\hline \multirow{2}{*}{ ETC } & $|\varepsilon|_{\text {avg }}(\mathrm{mV})$ & 13.8 & 11.2 & 13.4 \\
& $|\varepsilon|_{\max }(\%)$ & 1.76 & 1.35 & 1.65 \\
\multirow{2}{*}{ RETC } & $|\varepsilon|_{\text {avg }}(\mathrm{mV})$ & 15.6 & 12.6 & 13.6 \\
& $|\varepsilon|_{\max }(\%)$ & 1.77 & 1.61 & 1.68 \\
\hline
\end{tabular}

\subsection{Verification at Different Temperatures}

Furthermore, Fig. 11 shows experimental data and simulation results and at $-15^{\circ} \mathrm{C}$ and Table 5 lists simulation errors of two models under various temperatures, which are different from the temperatures used to obtain the models' parameters. The four different temperatures are obtained from 
battery temperatures when the battery is subjected to the SAC excitation of $10 \mathrm{~A}$ and $500 \mathrm{~Hz}$ for $1361 \mathrm{~s}$. Mean errors and maximum relative errors of presented models gradually increase with the decrease of temperature, indicating that the electrical behavior becomes more nonlinear at lower temperature. However, it can be concluded that the mean errors of ETC and RETC models are less than $16.18 \mathrm{mV}$ and $26.7 \mathrm{mV}$, respectively, and the maximum relative errors are less than $1.88 \%$ and $2.12 \%$ at four different temperatures, respectively. The well matched results confirm that simulation results of presented models are highly consistent with voltage profiles obtained from experiments, when the LIB is dynamically warmed up by SAC excitation in the environment of $-15^{\circ} \mathrm{C}$.
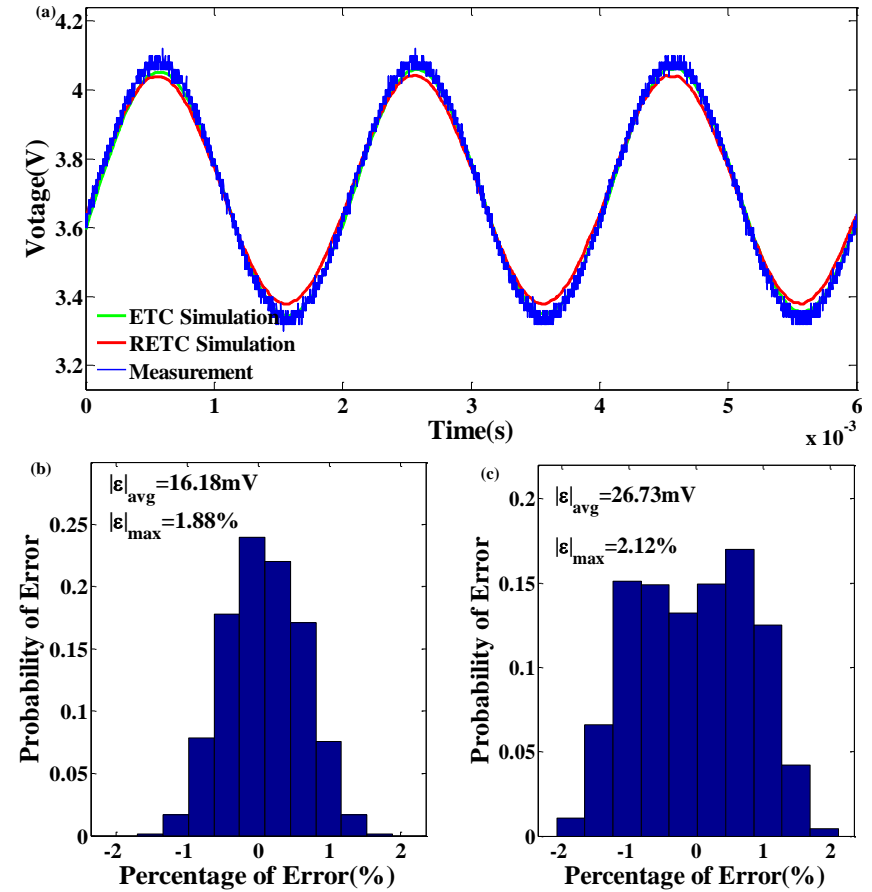

Fig.11. (a)Voltage evolution (b) Error distribution of ETC model simulation (c) Error distribution of RETC model simulation.

Table 5. Simulation errors of two models at $500 \mathrm{~Hz}$ under various temperatures

\begin{tabular}{cccccc}
\hline \multirow{2}{*}{ ETC } & Temperature $\left({ }^{\circ} \mathrm{C}\right)$ & -15 & -10 & -5 & 0.3 \\
\hline \multirow{2}{*}{ RETC } & $|\varepsilon|_{\text {avg }}(\mathrm{mV})$ & 16.2 & 12.6 & 12.0 & 11.0 \\
& $|\varepsilon|_{\max }(\%)$ & 1.88 & 1.64 & 1.52 & 1.51 \\
& $|\varepsilon|_{\text {avg }}(\mathrm{mV})$ & 26.7 & 18.4 & 17.0 & 12.4 \\
\hline
\end{tabular}

The temperature evolution and error distribution with $\mathrm{SAC}$ excitation of $10 \mathrm{~A}$ and $500 \mathrm{~Hz}$ for 1361s are shown in Fig.12. Obviously, there is a close match between battery temperatures extracted from experimental data and simulation results of ETC and RETC models, where the maximum absolute errors are less than $0.89^{\circ} \mathrm{C}$ and $1.58^{\circ} \mathrm{C}$, respectively. Furthermore, the battery is subjected to the different SAC excitation of CFCC, consisting of 50Hz-5A, and 5kHz-10A. The errors of two models, which are less than $1.5^{\circ} \mathrm{C}$ and $1.58^{\circ} \mathrm{C}$, respectively, confirm that models can predict 
accurately the thermal behavior of LIBs under various conditions, indicating the precision of temperature-dependent parameters from ETC and RETC models.
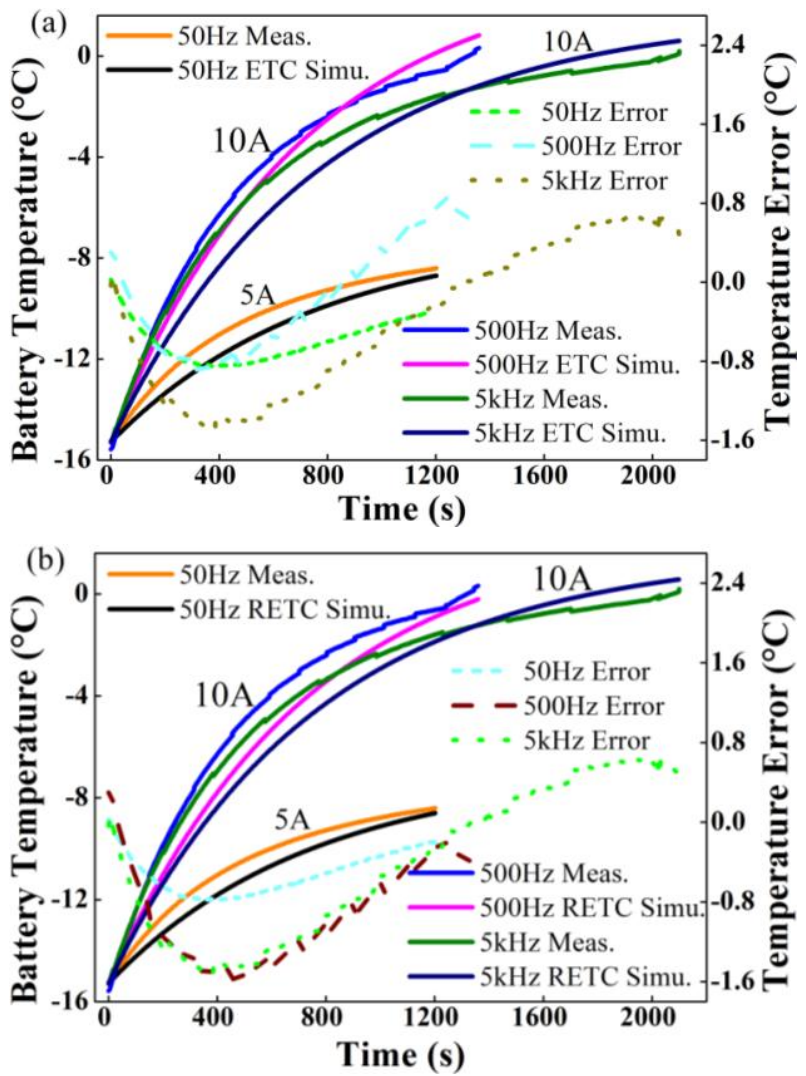

Fig.12. Temperature evolution and error distribution of (a) ETC model (b) RETC model at various frequencies.

\subsection{Verification with Different SAC Amplitudes}

We further verify the proposed models with steady-state SAC profiles of different amplitudes at 5kHz. Fig. 13 shows simulation results and measurements with the maximum SAC amplitude. Table 6 exhibits simulation errors of ETC and RETC models with current rates ranging from 0.07C to 3.64C. Good agreement can be observed for a variety of current rates at $-15^{\circ} \mathrm{C}$, where the mean errors are less than $16.2 \mathrm{mV}$ and $18.4 \mathrm{mV}$, respectively, and the maximum relative errors are less than $1.76 \%$ and $1.85 \%$, respectively. When the SAC amplitude is $10 \mathrm{~A}$, both mean error and maximum error are greater than those under other current rates. But the mean errors of presented models are kept the same and a low value, when the SAC amplitude becomes small, implying that it may be because of inaccuracy of the instrument.

When the battery is subjected to the SAC excitation of $500 \mathrm{~Hz}$ or $5 \mathrm{kHz}$ with several current amplitudes, the temperature results of measurement and simulation are shown in Fig. 14. Obviously, ETC and RETC models give promising results with the maximum error of $1.32^{\circ} \mathrm{C}$ and $1.79^{\circ} \mathrm{C}$ at CFVC conditions, respectively. Although the SAC amplitude varies, simulation temperatures' trends are nicely consistent with the measured ones, indicating that proposed models exhibit high accuracy 
in evaluating the battery temperature under various conditions.
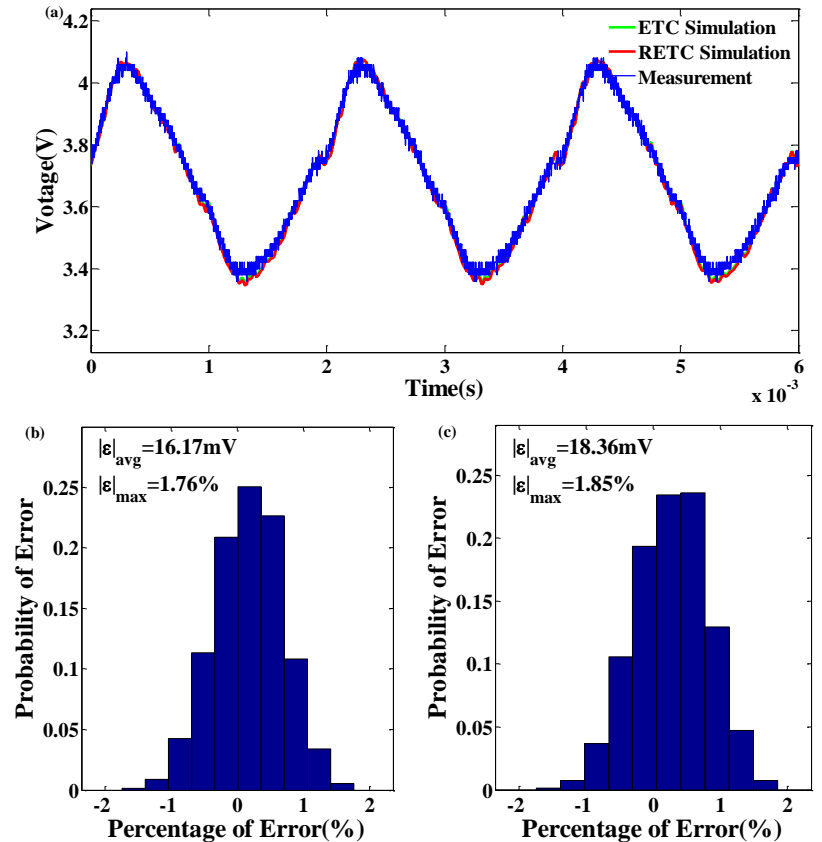

Fig.13. (a)Voltage evolution (b) Error distribution of ETC model simulation (c) Error distribution of RETC model simulation at $5 \mathrm{kHz}$ under $-15^{\circ} \mathrm{C}$

Table 6. Simulation errors of two models with various SAC amplitudes.

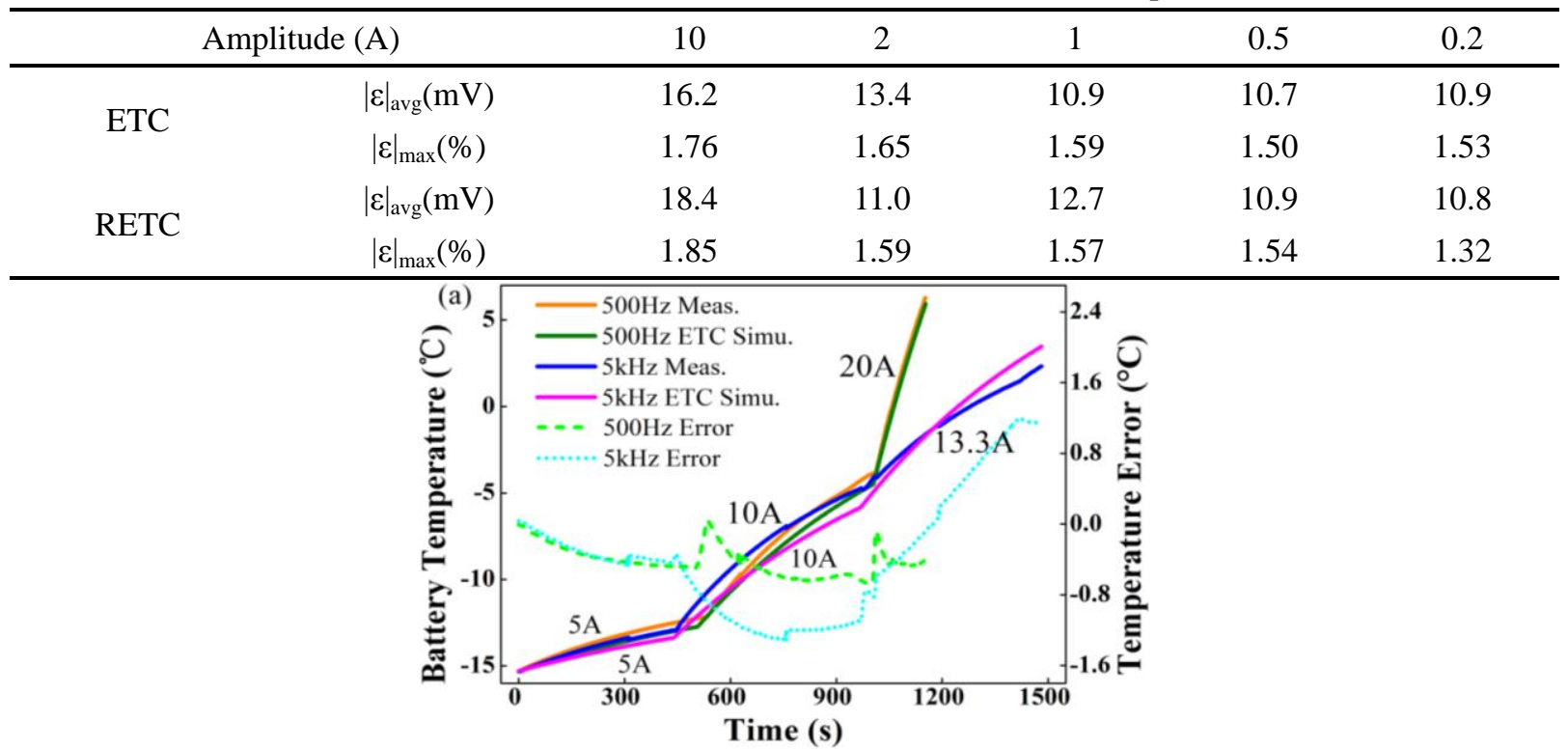

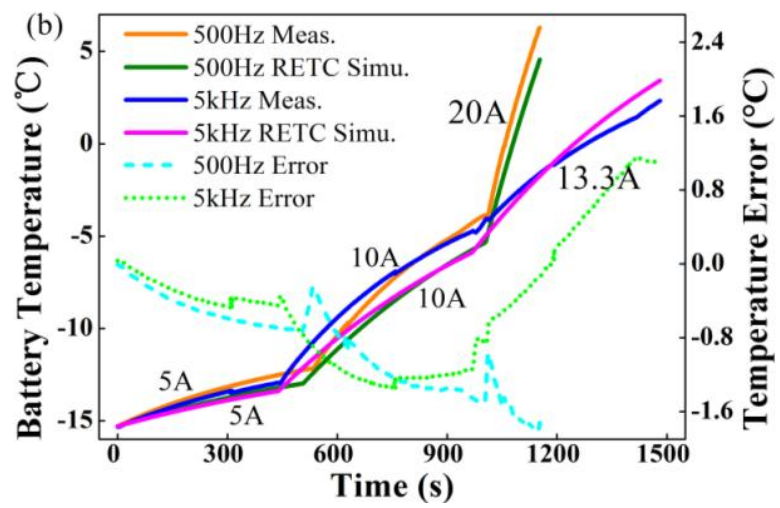

Fig.14. Temperature evolution and error distribution of (a) ETC model (b) RETC model with different SAC amplitudes. 
The simulation results of the RETC model represented by the mean and maximum relative voltage errors for all tests, where values are below $26.7 \mathrm{mV}$ and $2.12 \%$, respectively, confirm that the proposed model predicts the battery voltage with a higher accuracy. On the other hand, relying on the good results of the temperature-rise validation with the maximum error of below $1.79^{\circ} \mathrm{C}$, it is concluded that internal temperature-dependent parameters are also predicted correctly. The presented ETC model, which shows a little higher accuracy, contains more parameters and requires a higher computational effort. The proposed RETC model exhibits almost same accuracy with the ETC model under various conditions, but is free from the high computational effort, implying the great potential application in on-board BMS.

\subsection{Discussions}

\subsubsection{Model Comparison}

To better illustrate the advantage of the reduced electrical model, EEC, REEC, Thevenin I, Thevenin II model and the function, which is the exponential function as shown in Eq.(7), are taken to describe the impedance behavior, where the real part of impedance is taken as an example. The polarization resistance and capacitance in Thevenin I model is from the polarization within 1s while that in Thevenin II model is from the polarization within 0.1s. Fig.15 shows different simulation results and experimental data of the real impedance at $-15^{\circ} \mathrm{C}$. The simulation results of EEC, REEC model and the exponential function match the experimental data well. It is extremely difficult for the Thevenin model to describe the impedance behavior of LIBs.

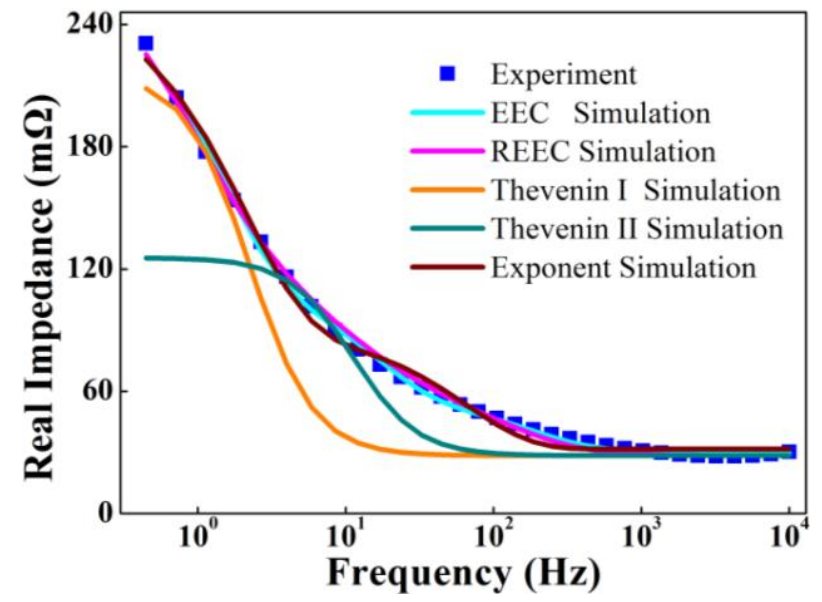

Fig.15. Real impedance from experiment and different simulation results.

$$
R_{e}=\lambda_{1} \cdot e^{-\frac{f}{\mu_{1}}}+\lambda_{2} \cdot e^{-\frac{f}{\mu_{2}}}+\kappa
$$

Simulation errors from Thevenin model are the highest as shown in Table 7. Thevenin model is not suitable for fitting the relationship between the real impedance and frequency whatever the 
polarization impedance is. Thevenin model can describe direct-current and certain-frequencyalternating-current behavior well, but has difficulty to predict the impedance behavior in wide frequency ranges. The minimum value of the maximum error is observed in the reduced model while the minimum mean error is seen in the EEC model. The accuracy of the reduced model is almost the same as EEC model in terms of impedance evaluation at different frequencies. However, there are twelve parameters needed to be used in EEC model, implying a higher computational effort. The reduced model, which is only composed of four parameters, requires significantly a lower computational effort.

Table 7. Simulation errors for the real impedance

\begin{tabular}{ccc}
\hline Errors & $|\varepsilon|_{\max }(\mathrm{m} \Omega)$ & $|\varepsilon|_{\text {avg }}(\mathrm{m} \Omega)$ \\
\hline EEC & 5.817 & 1.635 \\
REEC & 5.468 & 1.912 \\
Thevenin I & 49.457 & 16.454 \\
Thevenin II & 105.320 & 15.717 \\
Function & 7.910 & 3.591 \\
\hline
\end{tabular}

Simulation errors of the exponential function are small but there are ten parameters if the imaginary part of impedance is fitted. Furthermore, there only exists the mathematical relation if the impedance is fitted with the exponential function. Therefore, based on the FD equation, the simplified REEC model cannot only accurately describe electrochemical characteristic within the battery, but also precisely predict the impedance in wide frequency ranges with a lower computational effort.

\subsubsection{Adaptability of Reduced Model}

The simplified REEC model is not only used to describe the electrical behavior of NCM batteries, but also suitable for voltage prediction of other batteries from different manufacturers. The experiments of the LFP battery are carried out with steady-state SAC of wide frequency range from $50 \mathrm{~Hz}$ to $5 \mathrm{kHz}$, and high amplitude, from $2 \mathrm{C}$ to $9 \mathrm{C}$ as shown in Table 8 . It is concluded that the smaller the SAC amplitude is, the lower the simulation errors are. Simulation results of the reduced model show good agreements with experimental data under different conditions, where the highest values of maximum relative error and mean error are $2.18 \%$ and $17.3 \mathrm{mV}$, respectively.

Fig.16 exhibits the case of maximum error under different conditions, the error distribution of which is similar to that of NCM batteries. In addition, the reduced model for the LCO battery is validated with a high accuracy, where the maximum relative error and mean error are less than $2.09 \%$ and $18.1 \mathrm{mV}$, respectively. According to the highly consistent results between simulation and experiments using batteries with three different cathode materials from different manufacturers, the 
effectiveness and adaptability of the proposed methodology for model reduction is verified. It is also concluded that there is superior adaptability of the FD equation. Therefore, the reduced model yields a promising candidate for precise impedance evaluation of different batteries at various frequencies, and for development of low-temperature charging and self-heating strategies in on-board BMS.

Table 8. Simulation errors of the REEC model with SAC excitation

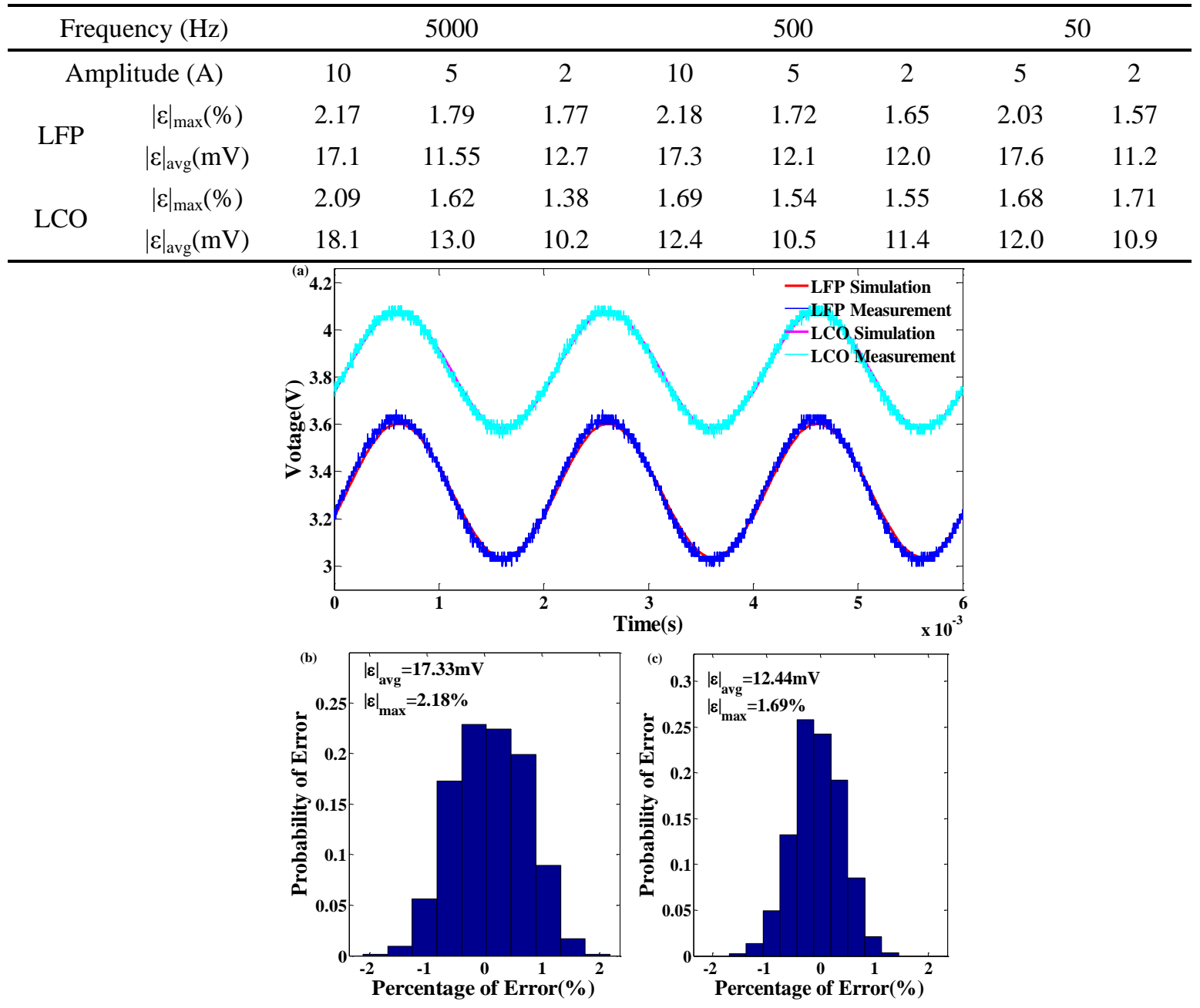

Fig.16. (a)Voltage evolution (b) Error distribution of simulation for LFP battery (c) Error distribution of simulation for LCO battery under $-15^{\circ} \mathrm{C}$ at $500 \mathrm{~Hz}$.

\subsubsection{Model Verification with UDDS Profile}

After the battery is charged with the current rate of $1 / 3 \mathrm{C}$ until the SOC reaches $51 \%$ at $-15^{\circ} \mathrm{C}$, it rests for 2 hours. The battery is subjected to the urban dynamometer driving schedule (UDDS) profile, in which the sampling time is $0.01 \mathrm{~s}$. The small fragment of the UDDS data segmented by a hamming window function is transformed via fast Fourier transformation (FFT). The main frequency, the amplitude of the current at which is high, is extracted and it is used as the frequency input of the RETC model. When the window is moved, another main frequency is obtained and used as the input of the proposed model again. Thus, the proposed RETC model can describe the electrical performance under the dynamically varying current profile. 
Fig. 17 shows the voltage evolution during simulation and experiment using the UDDS profiles. A comparison between experimental data and terminal voltage obtained from the battery simulation indicates that simulation results well match experimental data, where the mean voltage error and the maximum relative voltage error are $24.25 \mathrm{mV}$ and $2.65 \%$, respectively. Compared with sinusoidal current, the error under the UDDS condition slightly increases, which may result from the approximation of the selected main frequency or the variation of model parameters with SOC. However, the error is small enough for practical applications, indicating that the proposed model can accurately capture electrical performance under different operating conditions in cold weather.
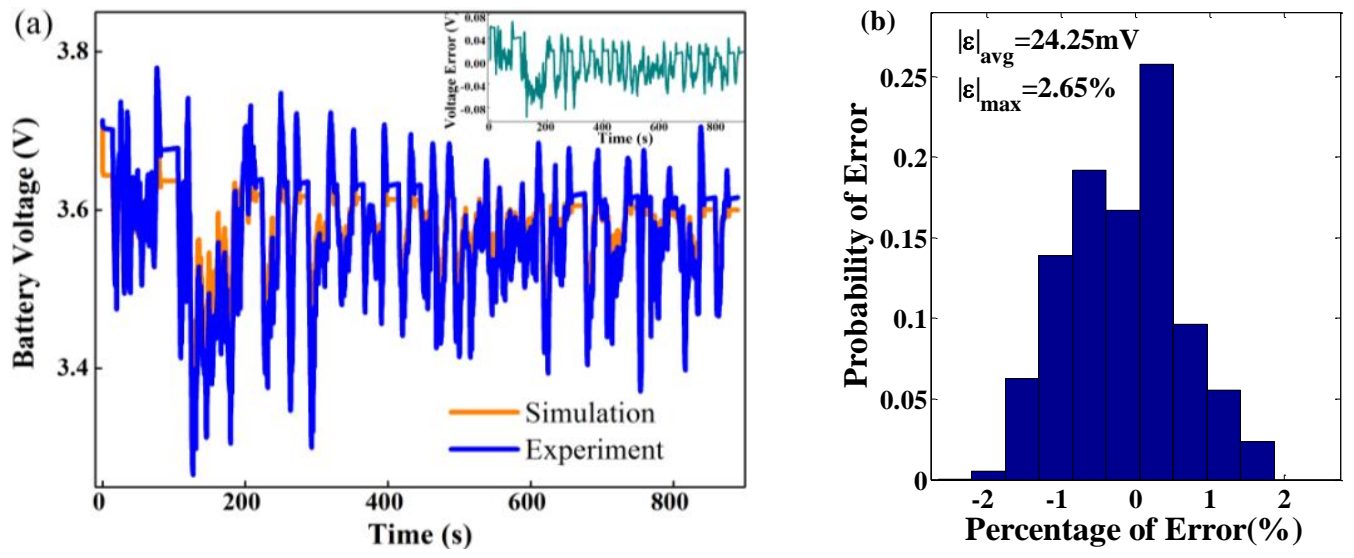

Fig. 17. (a) Voltage evolution during experiment and model simulation at $-15^{\circ} \mathrm{C}$, (b) Error distribution of the RETC model simulation.

Furthermore, the proposed reduced model can be used for other real applications with different dynamic profiles, using the similar process as described above for the UDDS profile. The reduced model, showing a high quality under different dynamical operation conditions, is of great potential for applications in on-board BMS and for simulation of powertrain systems in cold weather.

\section{Conclusions}

A simplified low-temperature RETC model for LIBs, which gives deep insight into both electrical and thermal performance, is developed with the following advantages:

(a) Simplified characteristic. Based on the strong influence of frequency on polarization voltage and irreversible heat, the frequency dependence of polarization resistance and capacitance is presented. With the help of new FD equation, the reduced model, which only contains four parameters, is simplified from the original sophisticated model.

(b) Temperature dependence. The simplified model, which can accurately predict voltage performance, is applied to describe low-temperature behavior of LIBs well. Based on the temperature-dependent parameters, the new model can precisely capture the temperature performance under different temperature, frequency and amplitude conditions. 
(c) High accuracy. Compared to the Thevenin model and the exponential function for impedance fitting, the reduced model exhibits a high accuracy as well as accurately characterizes electrochemical processes within batteries. This new model, which requires a lower computational effort, is demonstrated to have almost the same high accuracy as the sophisticated ETC model.

(d) Good adaptability. The effectiveness and adaptability of the proposed methodology for model reduction is verified based on the highly consistent results between simulation and experiments using batteries with three different cathode materials from different manufacturers. It is also concluded that there is superior adaptability of the new FD equation.

The proposed simple RETC model, with the maximum relative voltage error and temperature error of below $2.65 \%$ and $1.79^{\circ} \mathrm{C}$, respectively, can fulfill the requirement of engineering applications. The novel model, with lower computational effort and complexity under various conditions, yields a promising candidate for development of rapid charging and internal heating strategies at low temperature, for evaluation of LIB impedance at different frequencies, and for simulation of powertrain systems in cold weather. In addition, the parameters of the new model may be used to characterize the state of health of LIBs, implying the great potential for application in on-board BMS.

Further research is ongoing for implementing a general model, which takes the effect of the current and aging into account, resulting in high accuracy under complex conditions. The mechanism of how the SAC and DC affect the electrochemical reaction of LIBs is being investigated and their difference is being compared. In addition, the influence of SAC on battery lifetime, including SAC amplitude and frequency, is also being studied.

\section{Acknowledgements}

This work is supported by the National Science Foundation of China (Grant No. 51277010) and Postgraduate Innovation Foundation of Beijing Jiaotong University (Grant No. 2015YJS154). The authors would like to thank Professor Bor Yann Liaw from Hawaii Natural Energy Institute, SOEST, University of Hawaii for his contribution to the overall structure and thank Professor Barry Morris from Hanergy School of Renewable Energy, Beijing Jiaotong University for the language improvement of the main content.

\section{Appendix}

Table 9. Fitting results of electrical parameters in ETC model

\begin{tabular}{ccccccccccccc}
\hline & $\begin{array}{c}R_{f} \\
(\mathrm{~m} \Omega)\end{array}$ & $\begin{array}{c}C_{f} \\
(\mathrm{mF})\end{array}$ & $\begin{array}{c}R_{a} \\
(\mathrm{~m} \Omega)\end{array}$ & $\begin{array}{c}C_{a} \\
(\mathrm{mF})\end{array}$ & $\begin{array}{c}R_{c} \\
(\mathrm{~m} \Omega)\end{array}$ & $\begin{array}{c}C_{c} \\
(\mathrm{mF})\end{array}$ & $\begin{array}{c}R_{d 1} \\
(\mathrm{~m} \Omega)\end{array}$ & $\begin{array}{c}C_{d 1} \\
(\mathrm{mF})\end{array}$ & $\begin{array}{c}R_{d 2} \\
(\mathrm{~m} \Omega)\end{array}$ & $\begin{array}{c}C_{d 2} \\
(\mathrm{mF})\end{array}$ & $\begin{array}{c}R_{b} \\
(\mathrm{~m} \Omega)\end{array}$ & $\begin{array}{c}R_{f} \\
(\mathrm{~m} \Omega)\end{array}$ \\
\hline Intercept & -10.91 & 13.39 & -13.98 & 9.47 & -20.48 & 10.81 & -26.85 & 19.88 & -3.60 & 25.84 & 0.90 & -10.91 \\
Slope & 3.52 & -2.60 & 4.50 & -1.19 & 6.43 & -1.19 & 8.17 & -3.13 & 2.02 & -3.51 & 0.63 & 3.52 \\
\hline
\end{tabular}


Table 10. Fitting results of electrical parameters in RETC model

\begin{tabular}{ccccccc}
\hline & $R_{\alpha}(\Omega)$ & $C_{\alpha}(\mathrm{F})$ & $R_{\beta}(\Omega)$ & $C_{\beta}(\mathrm{F})$ & $R_{\gamma}(\Omega)$ & $C_{\gamma}(\mathrm{F})$ \\
\hline Intercept & -6.26 & 6.38 & 23.05 & 2.24 & -15.87 & 5.80 \\
Slope & 1.58 & -1.64 & -5.58 & -0.45 & 3.16 & -2.38 \\
\hline
\end{tabular}

Table 11. Fitting results of electrical parameters in RETC model with different batteries

\begin{tabular}{ccccccccc}
\hline Battery & $R_{\alpha}(\Omega)$ & $C_{\alpha}(\mathrm{F})$ & $R_{\beta}(\Omega)$ & $C_{\beta}(\mathrm{F})$ & $R_{\gamma}(\Omega)$ & $C_{\gamma}(\mathrm{F})$ & $R_{b}(\Omega)$ & $L(\mathrm{nH})$ \\
\hline LFP & 4.255 & 4.622 & 0.0165 & 1.934 & 15.516 & 0.020 & 0.0210 & 100.563 \\
LCO & 1.298 & 2.806 & 0.0197 & 2.291 & 6.937 & 0.0232 & 0.0184 & 118.848 \\
\hline
\end{tabular}

\section{References}

[1] Y. Xing, H. Wei, M. Pecht, et al. State of charge estimation of lithium-ion batteries using the open-circuit voltage at various ambient temperatures [J]. Applied Energy, 2014, 113(1):106-115.

[2] W. Waag, S. Käbitz, DU. Sauer. Experimental investigation of the lithium-ion battery impedance characteristic at various conditions and aging states and its influence on the application [J]. Applied Energy, 2013, 102(2):885-897.

[3] Fan J, Tan S, Fan J, et al. Studies on Charging Lithium-Ion Cells at Low Temperatures [J]. Journal of the Electrochemical Society, 2006, 153(6):A1081-A1092.

[4] T. A. Stuart, A. Hande. HEV battery heating using AC currents [J]. Journal of Power Sources, 2004, 129(2):368-378.

[5] T. Wang, K. J. Tseng, J. Zhao, et al. Thermal investigation of lithium-ion battery module with different cell arrangement structures and forced air-cooling strategies [J]. Applied Energy, 2014, 134(C):229-238.

[6] P. Ping, Q. Wang, P. Huang, et al. Thermal behaviour analysis of lithium-ion battery at elevated temperature using deconvolution method [J]. Applied Energy, 2014, 129:261-273.

[7] Zhao X W, Zhang G Y, Yang L, et al. A new charging mode of Li-ion batteries with $\mathrm{LiFePO}_{4} / \mathrm{C}_{\text {composites }}$ under low temperature [J]. Journal of Thermal Analysis \& Calorimetry, 2011, 104(2):561-567.

[8] Salkind A J, Fennie C, Singh P, et al. Determination of state-of-charge and state-of-health of batteries by fuzzy logic methodology [J]. Journal of Power Sources, 1999, 80(s 1-2):293-300.

[9] CC. Chan, EWC. Lo, W. Shen. The available capacity computation model based on artificial neural network for lead-acid batteries in electric vehicles [J]. Journal of Power Sources, 2000, 87(s 1-2):201-204.

[10] T. R. Tanim, C. D. Rahn, C. Y. Wang. State of charge estimation of a lithium ion cell based on a temperature dependent and electrolyte enhanced single particle model [J]. Energy, 2015, 80:731-739.

[11] M. Guo, G. H. Kim, R. E. White. A three-dimensional multi-physics model for a Li-ion battery [J]. Journal of Power Sources, 2013, 240(1):80-94.

[12] S. Liu, J. Jiang, W, Shi, et al. Butler-volmer-equation-based electrical model for high-power lithium titanate batteries used in electric vehicles [J]. IEEE Transactions on Industrial Electronics, 2015, 62: 7557-7568.

[13] W. Y. Low, J. A. Aziz, N. R. N. Idris, et al. Electrical model to predict current-voltage behaviours of lithium ferro phosphate batteries using a transient response correction method [J]. Journal of Power Sources, 2013, 221(1):201-209.

[14] X. Hu, S. Li, H. Peng, et al. Charging time and loss optimization for $\mathrm{LiNMC}$ and $\mathrm{LiFePO}_{4}$ batteries based on equivalent circuit models[J]. Journal of Power Sources, 2013, 239(10):449-457.

[15] J. Jiang, Q. Liu, C. Zhang, et al. Evaluation of Acceptable Charging Current of Power Li-Ion Batteries Based on Polarization Characteristics [J]. IEEE Transactions on Industrial Electronics, 2014, 61(12):6844-6851.

[16] T. K. Dong, A. Kirchev, F. Mattera, et al. Dynamic Modeling of Li-Ion Batteries Using an Equivalent Electrical Circuit [J]. Journal of the Electrochemical Society, 2011, 158(3):A326-A336.

[17] X. Hu, S. Li, H. Peng. A comparative study of equivalent circuit models for Li-ion batteries [J]. Journal of 
Power Sources, 2012, 198(198):359-367.

[18] M. Montaru, S. Pelissier. Frequency and Temporal Identification of a Li-ion Polymer Battery Model Using Fractional Impedance [J]. Oil \& Gas Science \& Technology-Revue De L'institut Français Du Pétrole, 2010, 65(1):67-78.

[19] D. Andre, M. Meiler, K. Steiner, et al. Characterization of high-power lithium-ion batteries by electrochemical impedance spectroscopy. II: Modelling [J]. Journal of Power Sources, 2011, 196(12):5349-5356.

[20] A. Lasia. Electrochemical impedance spectroscopy and its applications [J]. Modern Aspects of Electrochemistry, 1999, 32(4):143-248.

[21] H. M. Cho, W. S. Choi, J. Y. Go, et al. A study on time-dependent low temperature power performance of a lithium-ion battery[J]. Journal of Power Sources, 2012, 198(1):273-280.

[22] T. Tanaka, S. Ito, M. Muramatsu, et al. Accurate and versatile simulation of transient voltage profile of lithium-ion secondary battery employing internal equivalent electric circuit [J]. Applied Energy, 2015, 143:200-210.

[23] L. Zhang, X. Hu, Z. Wang, et al. Experimental impedance investigation of an ultracapacitor at different conditions for electric vehicle applications[J]. Journal of Power Sources, 2015, 287:129-138.

[24] Tippmann S, Walper D, Balboa L, et al. Low-temperature charging of lithium-ion cells part I: Electrochemical modeling and experimental investigation of degradation behavior [J]. Journal of Power Sources, 2014, 252:305-316.

[25] Yi J, Kim U S, Shin C B, et al. Modeling the temperature dependence of the discharge behavior of a lithium-ion battery in low environmental temperature [J]. Journal of Power Sources, 2013, 244:143-148.

[26] Remmlinger J, Tippmann S, Buchholz M, et al. Low-temperature charging of lithium-ion cells Part II: Model reduction and application [J]. Journal of Power Sources, 2014, 254(15):268-276.

[27] J. P. Schmidt, T. Chrobak, M. Ender, et al. Studies on LiFePO4 as cathode material using impedance spectroscopy [J]. Journal of Power Sources, 2011, 196(12):5342-5348.

[28] D. P. Abraham, A. N. Jansen, Heaton J R, et al. Investigating the Low-Temperature Impedance Increase of Lithium-Ion Cells[J]. Journal of the Electrochemical Society, 2008, 155(1):A41-A47.

[29] C. A. Schiller, F. Richter, E. Gülzow, et al. Relaxation impedance as a model for the deactivation mechanism of fuel cells due to carbon monoxide poisoning [J]. Phys.chem.chem.phys, 2001, 3(11):2113-2116.

[30] S. Arrhenius. The reaction velocity of the inversion of cane sugar by acids [J]. Selected Readings in Chemical Kinetics, 1967:31-35.

[31] D. Bernardi, E. Pawlikowski, J. Newman. A General Energy Balance for Battery Systems [J]. Journal of the Electrochemical Society, 1984, 132(1):5-12.

[32] C. T. O'Sullivan. Newton's law of cooling-A critical assessment [J]. American Journal of Physics, 1990, 58(10):956-960.

[33] C. Forgez, D. V. Do, G. Friedrich, et al. Thermal modeling of a cylindrical LiFePO 4/graphite lithium-ion battery[J]. Journal of Power Sources, 2010, 195(9):2961-2968.

[34] M. A. Monem, K. Trad, N. Omar, et al. Lithium-ion batteries: Evaluation study of different charging methodologies based on aging process [J]. Applied Energy, 2015, 152:143-155.

[35] E. Barsoukov, R. J. Macdonald. Impedance spectroscopy: Theory, experiment, and applications[J]. Journal of Hospice \& Palliative Nursing, 2005, 4(4):206-207. 

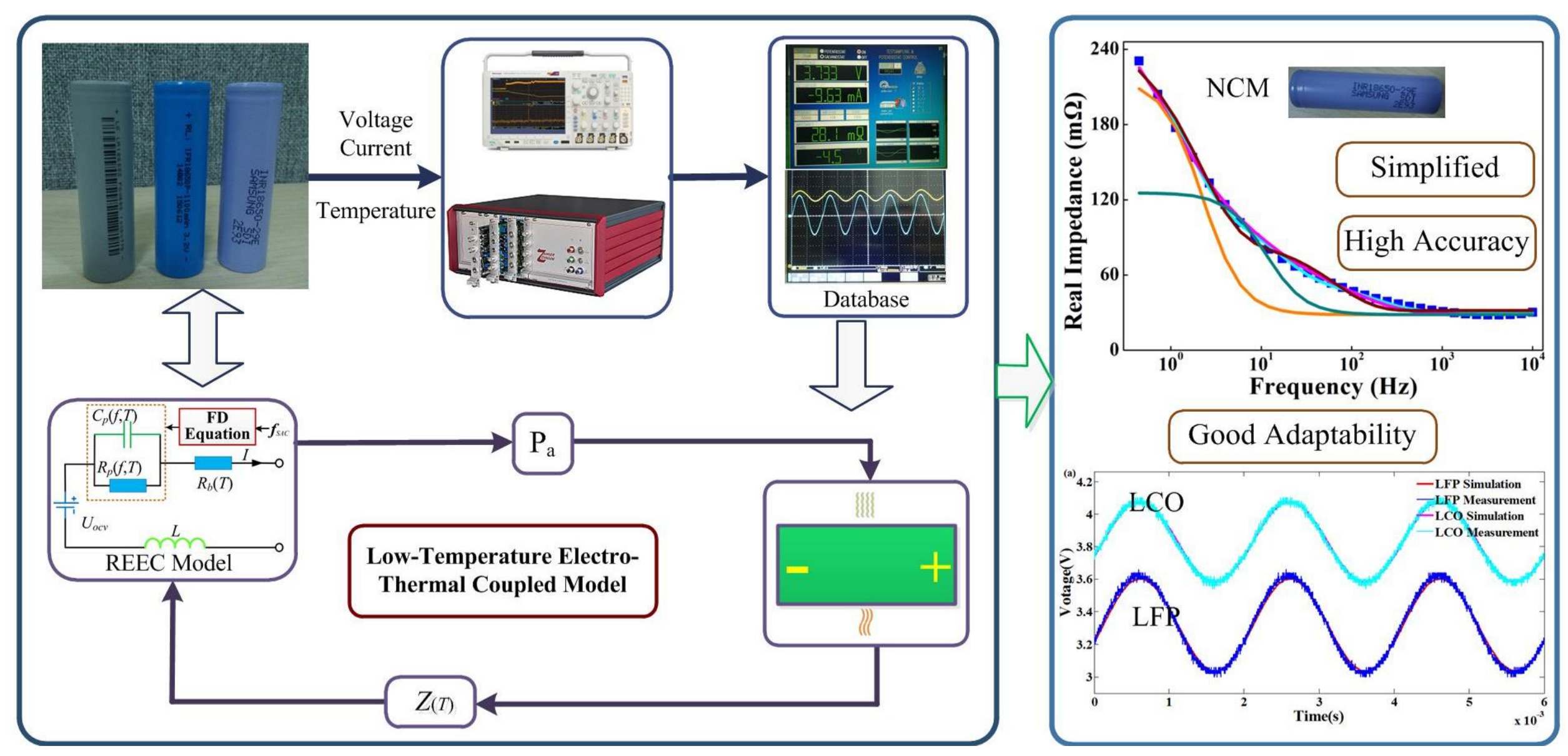